Arribas-Galarraga, S. \& Maiztegi-Kortabarria, J. (2021). Evolución de la atención, concentración y rendimiento académico tras una intervención basada en descansos activos. Revista Electrónica Interuniversitaria de Formación del Profesorado, 24(3), 87-100.

DOI: https://doi.org/10.6018/reifop.467731

\title{
Evolución de la atención, concentración y rendimiento académico tras una intervención basada en descansos activos
}

Silvia Arribas-Galarraga, Julen Maiztegi-Kortabarria

Universidad del País Vasco - Donostia-San Sebastian, España.

\section{Resumen}

El objetivo principal de la investigación ha sido analizar la evolución de la atención y concentración en jóvenes estudiantes, tras la implementación de un programa basado en descansos activos o Activity Breaks en la asignatura de Lengua Castellana y Literatura. La muestra, compuesta por 31 estudiantes, 14 chicas y 17 chicos, del primer curso de la Enseñanza Secundaria Obligatoria (edad media 12.13 años), se dividió en dos grupo clase: grupo control y grupo experimental. La intervención, de 7 semanas, implementada durante las lecciones en el aula, permitió aumentar la cantidad de actividad física diaria. Mediante el instrumento de medida "Test de atención D2", se midió la atención y la concentración, y a través de un texto dictado el rendimiento ortográfico, los datos se recogieron tanto en la fase previa, como tras la intervención. Los resultados indican un aumento estadísticamente significativo tanto en la variable atención como en la variable concentración en el grupo experimental. En cuanto a los contenidos relativos a Lengua Castellana y Literatura, ortografía, no se observaron diferencias estadísticamente significativas.

\section{Palabras clave}

Descansos activos; atención; concentración; rendimiento académico.

\section{Contacto:}

Julen Maiztegi Kortabarria, jmaiztegi@ikasle.ehu.eus, Didáctica de la Expresión Musical, Plástica y Corporal, Universidad del País Vasco (UPV-EHU). 


\title{
Evolution of attention, concentration and academic performance after an intervention based on Activity Breaks
}

\begin{abstract}
The main objective of the research has been to study the evolution of attention and concentration after the implementation of a programme based on Activity Breaks in the subject Spanish Language and Literature. The sample, formed by 31 students, 14 girls and 17 boys, from the first course o Compulsory Secondary Education (average age 12.13 years), was divided into two class groups, control group and experimental group. The intervention, of 7 weeks, implemented during the lessons in the classroom, allowed the amount of daily physical activity to be increased. By the measurement instrument "Attention Test D2" attention and concentration and by the text dictated spelling performance was measured, data was collected in the previous phase and after the intervention. The results indicate a statically significant increase in attention and concentration variable in experiential group. Regarding the Spanish Language and Literature contents, orthography, no statistically significant differences were observed.
\end{abstract}

\section{Keywords}

Active Breaks; attention; concentration; academic performance.

\section{Introducción}

Desde mediados del siglo XX hasta la actualidad los hábitos socio-laborales de las culturas más avanzadas han evolucionado en detrimento de la actividad física. El aumento del uso de medios de transporte mecanizados y las técnicas cada vez más modernas de trabajo, han conducido a la población hacia actividades con un reducido gasto energético catalogadas como sedentarias (Arocha Rodulfo, 2019; Romero, 2009; Tremblay et al., 2011). Uno de los informes más recientes de la Organización Mundial de la Salud (OMS, 2020), indica que los niveles de actividad físico-deportiva de la población mundial son insuficientes, tanto en la población adulta, como en la población infantil y adolescente (García Maramoros, 2019). Entre los argumentos que se adoptan para afirmar, que la población más joven, tiene escasas oportunidades para mantenerse activa, Mahar (2011), apunta la escasez de espacios de juego, el aumento del tiempo dedicado al uso de dispositivos electrónicos y un aumento de la exigencia de escolarización. De acuerdo a los datos del Ministerio de Sanidad, Consumo y Bienestar Social (2017), el 73.9 \% de las niñas y niños de entre 1 a 14 años de edad, emplea una hora o más de su tiempo libre al uso de pantallas entre semana, aumentando las cifras los fines de semana hasta 82.6\%. Diversos autores (Arocha Rodulfo, 2019; Carson et al., 2016) coinciden en afirmar que los hábitos orientados a actitudes más sedentarias e inactividad físico-deportiva, repercuten de distinta manera sobre la salud, pudiendo derivar en enfermedades crónicas y en resultados fisiológicos, cognitivos y de comportamiento negativo (Glapa et al., 2018).

La evidencia científica muestra cierta asociación entre la práctica de activad físico-deportiva y la salud. En este sentido, la revisión sistemática realizada por Timmons et al. (2012) revela que la práctica de actividad física durante los primeros años puede incidir en el estado futuro de la salud. Sin embargo, más allá de la salud física, la actividad físico-deportiva incide de forma integral en el bienestar de las personas (Ahumada Tello y Toffoletto, 2020). El trabajo de Poitras et al. (2016), relaciona positivamente este tipo de actividad con variables 
referentes tanto a la salud física, como a la social y cognitiva de niñas, niños y adolescentes, indicando beneficios, principalmente cuando las actividades físico-deportivas son de mayor intensidad, en la calidad de vida, bienestar, y el desarrollo de las habilidades motoras entre otras.

En esta línea, diversos autores coinciden en afirmar en la necesidad de la promoción y el mantenimiento de la actividad físico-deportiva como un objetivo global, para mejorar la salud, la aptitud física y la regulación del peso corporal saludable en niñas, niños y adolescentes (Bernate, J. A., Fonseca y Cruz, 2020; Robinson et al., 2015). Además, insisten en reducir los tiempos prolongados en situación sedentaria (Webster, Russ, Vazou, Goh y Erwin, 2015). La conducta sedentaria se define como la carencia de movimiento durante las horas de vigilia a lo largo del día (Tremblay et al., 2010). Actividades como ver la televisión y estar acostado o sentado durante largos periodos de tiempo, son claro ejemplo de actitudes sedentarias.

Los y las jóvenes estudiantes pasan en la escuela numerosas horas a lo largo del día (Mahar et al., 2006), siendo que el $80 \%$ del tiempo lo pasan sentados o realizando actividades sedentarias (Sánchez, Gutiérrez, Ruiz de la Hermosa, López, y Sánchez, 2017). Autores como Marchesi y Pérez (2003) o Rodríguez Torres, et al. (2020), destacan que la eficacia y éxito de las escuelas, dependen de los rasgos que definan al centro escolar, así como del esfuerzo compartido por toda la comunidad educativa. Por todo ello, se podría considerar el contexto escolar como una excelente oportunidad para promocionar la actividad física e impulsar la salud (Webster et al., 2015), por ejemplo, los recreos, las actividades extraescolares, los trayectos a la escuela y a casa, la asignatura de educación física o incluso el introducir actividad física dentro del aula como indica Méndez-Giménez (2020).

Existen estudios que abordan aumentar la práctica de actividad físico-deportiva reduciendo los periodos prolongados sedentarios del alumnado en el aula: los descansos activos (Layne, Yli-Piipari y Knox, 2020; Méndez-Giménez, 2020; Merriman, González-Toro y Cherubini, 2020). Los descansos activos o Activity Breaks, consisten en la práctica de actividad físico-deportiva de intensidad moderada o vigorosa con una duración de entre 5 a 10 minutos, durante la lección académica (Masini et al., 2020). Las investigaciones indican, que este tipo de intervención aumenta los niveles de actividad física realizada por el alumnado, además de mejorar la salud de los y las jóvenes (Abi, Hilberg, Schuna, John y Gunter, 2018). También, proporciona beneficios de aprendizaje: motivación, disfrute del aprendizaje, disminución de los problemas de comportamiento, y mejoras en la atención, el comportamiento en la tarea y el rendimiento académico (Merriman et al., 2020). Según la revisión sistemática y metaanálisis de Álvarez-Bueno et al. (2017), en la que analizaron el efecto de las intervenciones de actividad física en las y los estudiantes con edades comprendidas entre los 4 y 18 años, concluyeron que la práctica de actividad físico-deportiva, tanto dentro del aula como fuera, fomenta el desarrollo de la cognición. Por su parte, Pesce et al. (2009), descubrieron que la práctica de actividad físico-deportiva produce cambios transitorios de comportamiento y psicológicos, que reflejan una modulación de la actividad de las redes neuronales, produciendo cambios en el nivel de activación y en los procesos cognitivos responsables de la asignación de los recursos mentales. No obstante, Tomporowski, McCullick, Pendleton y Pesce (2015) señalan que el nexo entre la cognición y el rendimiento académico es complejo, y posiblemente influyen otros factores. De este modo, diversos autores recalcan el rendimiento académico como una dimensión multifactorial (Kapur, 2018; Willcox, 2011; Torres y Rodríguez, 2006; Zuluaga, 2017; Lamas, 2015).

Una de las funciones atribuidas a la escuela, es impulsar y desarrollar la comprensión y aprendizaje del alumnado. A este efecto, variables como la atención y concentración resultan relevantes (Vanhelst et al., 2016). La atención es una función neuropsicológica compleja, 
compuesta por diferentes mecanismos que trabajan de forma coordinada, con la finalidad de seleccionar y filtrar del entorno los estímulos importantes para ejecutar una acción concreta (Ríos-Lago, Muñoz-Céspedes y Paúl-Lapedriza, 2007). La concentración, es la capacidad de mantener la atención en una tarea durante un cierto tiempo, mientras se ejecuta un proceso mental especifico o tarea concreta (Ocampo, 2009). La revisión realizada por Maureira y Flores (2017), señala que la atención se mejora tras la práctica de actividad físico-deportiva.

En cuanto a las investigaciones que abordan los descansos activos o Activity Breaks, se encuentra el estudio de Muñoz-Parreño, Belando-Pedreño, Torres-Luque y Valero-Valenzuela (2020), en el que se observó que los descansos activos contribuyeron a reducir los periodos sedentarios y a aumentar los niveles de actividad física moderada y vigorosa de acuerdo a las recomendaciones sobre la cantidad de actividad necesaria diaria. La revisión sistemática sobre los descansos activos realizado por Masini et al. (2020) muestra que la implementación de actividad físico-deportiva dentro del aula, aumenta de manera significativa el tiempo dedicado a la actividad física de intensidad moderada o vigorosa, presentando mejoría en el comportamiento del alumnado. Los resultados en cuanto a las funciones cognitivas no fueron concluyentes. Por su parte, Contreras-Jordán, León, Infantes-Paniagua y Prieto-Ayuso (2020), demostraron la relación significativa existente entre la implementación de programas basados en descansos activos, con la mejora de la concentración y la atención selectiva. Concluyeron que el incremento de las funciones cognitivas propicia en el alumnado, un mejor aprovechamiento de las clases, mayor motivación y por ello, mejora en el rendimiento cognitivo y académico. De igual modo, los resultados del estudio de Pinto-Escalona y Martínez-de-Quel (2019) indicaron que la práctica de actividad física realizada durante 10 minutos en la asignatura de Lengua Castellana y Literatura, mejoraba de manera significativa la atención, la cantidad de actividad física realizada y el rendimiento académico. Asimismo, Ruiz-Ariza, López-Serrano, Mezcua-Hidalgo, Martínez-López y Abu-Helaiel (2020), analizaron la incidencia y el efecto de los descansos activos de baja y alta intensidad de 136 adolescentes de entre 12 y 14 años de edad, concluyendo que los descansos activos de baja intensidad producen una mejora del 21 al 25 \% en las variables de atención y concentración. En la misma línea, Janssen et al. (2014), en su investigación, descubrieron un efecto significativo de los descansos activos sobre la atención selectiva de los y las jóvenes de 10 y 11 años de edad, obteniendo mejores resultados en las intervenciones de intensidad moderada.

Así pues, algunos autores afirman (Muñoz-Parreño et al., 2020) que la implementación de actividad física dentro del aula, integrada en el currículum, reduce los niveles de sedentarismo. Además, el incremento de los niveles de la práctica de actividad física a intensidad moderada y vigorosa (Masini et al., 2020), permite acumular la cantidad recomendada por la OMS (2020), es decir, practicar como mínimo 60 minutos de actividad física-deportiva con una intensidad moderada o intensa. $Y$ realizar actividades dirigidas a fortalecer los músculos y los huesos, al menos tres veces a la semana, para la población infantil y adolescente con edades comprendidas entre los 5 y 17 años, permite promover un gasto significativo de energía entre el alumnado (Stewart, Dennison, Kohl y Doyle, 2004). A su vez, este tipo de actividades, ayudarían a aumentar los niveles de las variables relacionadas con el aprendizaje como la concentración, atención, la cognición y el tiempo en tarea (Méndez-Giménez, 2020; Ruiz-Ariza et al., 2020).

La literatura científica revisada, informa por un lado sobre las numerosas horas que a lo largo del día los jóvenes están en la escuela (Mahar et al., 2006) y por otro, del incremento de los niveles de sedentarismo y/o inactividad (OMS, 2020). Del mismo modo, se advierten los beneficios de la actividad física a intensidad moderada y vigorosa (Muñoz-Parreño et al., 2020) no solo en la salud física, sino también sobre aspectos relacionados con el rendimiento académico. El presente trabajo, toma la escuela como contexto para estudiar la incidencia de 
la implementación de un programa de Activity Breaks en la asignatura de Lengua Castellana y Literatura, en la evolución de la atención y concentración en adolescentes. Los objetivos específicos se centran en: 1) Analizar y comprobar la evolución del nivel de atención y concentración del alumnado en función del programa de descansos activos; 2) Examinar la relación existente entre el rendimiento académico y los descansos activos.

\section{Método}

\section{Participantes}

La muestra, de tipo incidental, está constituida por estudiantes del primer curso de la Enseñanza Secundaria Obligatoria (ESO), que cursan la asignatura Lengua Castellana y Literatura en un centro escolar de la Comunidad Autónoma del País Vasco. Participaron un total de 31 alumnos y alumnas de entre 12-13 años $(M=12.13$, DT $=0.341)$, de los cuales 14 eran chicas ( $45.16 \%$ ) y 17 chicos ( $54.84 \%)$. La muestra se dividió en función de los grupos clase actuando uno como grupo control $(n=14)$ y el otro como grupo intervención $(n=17)$.

\section{Instrumentos}

Test de Atención D2 (Brickenkamp, 2002). Se utilizó la versión española de Cubero (2012). Este test mide la atención selectiva y la concentración mental, de modo individual y colectivo, de la población comprendida entre 8-88 años de edad. El Test D2 está formado por 14 líneas compuestas por 47 caracteres, 658 en total, representadas por las letras " $d$ " o " $p$ ". Cada letra puede contener una o dos rayas situadas en pareja o individualmente, en la parte superior 0 inferior. El test se basa en revisar el contenido de cada línea en un tiempo estipulado de 20 segundos de izquierda a derecha y señalar toda letra "d" que contenga dos rayas, es decir, los elementos relevantes. Las puntuaciones resultantes son: TR, total de respuestas, números de elementos intentados en las 14 líneas; TA, total de aciertos, número de elementos relevantes correctos; $\mathrm{O}$, omisiones, número de elementos relevantes intentados pero no marcados; $C$, comisiones, número de elementos irrelevantes marcados; TOT, efectividad total en la prueba, es decir, TR - $(\mathrm{O}+\mathrm{C})$; CON, índice de concentración o TA - C; $\mathrm{TR}+$, línea con mayor número de elementos intentados; TR -, línea con menor número de elementos intentados y VAR, índice de variación o diferencia (TR+) - (TR-). La duración estimada para su cumplimentación, incluida las instrucciones de aplicación, es de 8-10 minutos.

La versión española presenta una fiabilidad alta de las dimensiones principales (TR, TA, O y C), siendo que los coeficientes de fiabilidad oscilan desde .86 hasta .99 .

Dictado. Texto previamente acordado con el profesorado para recoger los datos referentes al rendimiento académico en la asignatura de Lengua Castellana y Literatura. A la hora de corregir, se tuvo en cuenta el contenido trabajado mediante la intervención de Activity Breaks, en este caso, el número de errores cometidos respecto a las tildes.

\section{Procedimiento y Programa de Intervención}

En primera instancia se diseñó el protocolo de actuación respetando la normativa establecida por la Comisión Ética para la Investigación con Seres Humanos de la Universidad, así como lo dispuesto para el tratamiento de los datos de carácter personal (Ley Orgánica 3/2018, de 5 de diciembre, de Protección de Datos Personales y garantía de los derechos digitales). La 
participación fue voluntaria y confidencial y se obtuvo el consentimiento informado por parte de los participantes antes de la administración de los cuestionarios.

Tras el primer contactó con el centro participante en la investigación, se efectuaron reuniones con la dirección y con la profesora que impartiría la asignatura Lengua Castellana y Literatura en $1^{\circ}$ de ESO (Educación Secundaria Obligatoria), y quién sería la encargada de dirigir los descansos activos en las sesiones de aula. Una vez obtenidas la autorización del centro y de las familias, el alumnado participante firmó el consentimiento informado. La recogida de datos se realizó en el aula.

La muestra se seleccionó por conveniencia, teniendo en cuenta la disposición del profesorado que impartiera clase de la misma asignatura a dos grupos o más, del mismo nivel educativo. Los grupos control y experimental se determinaron mediante sorteo.

Paralelamente, se fue diseñando un fichero con actividades para realizar la intervención de descansos activos o Activity Breaks, que al tiempo, permiten trabajar y repasar contenidos relacionados con la ortografía y redacción.

Figura 1.

Ficha explicativa para el profesorado de aula

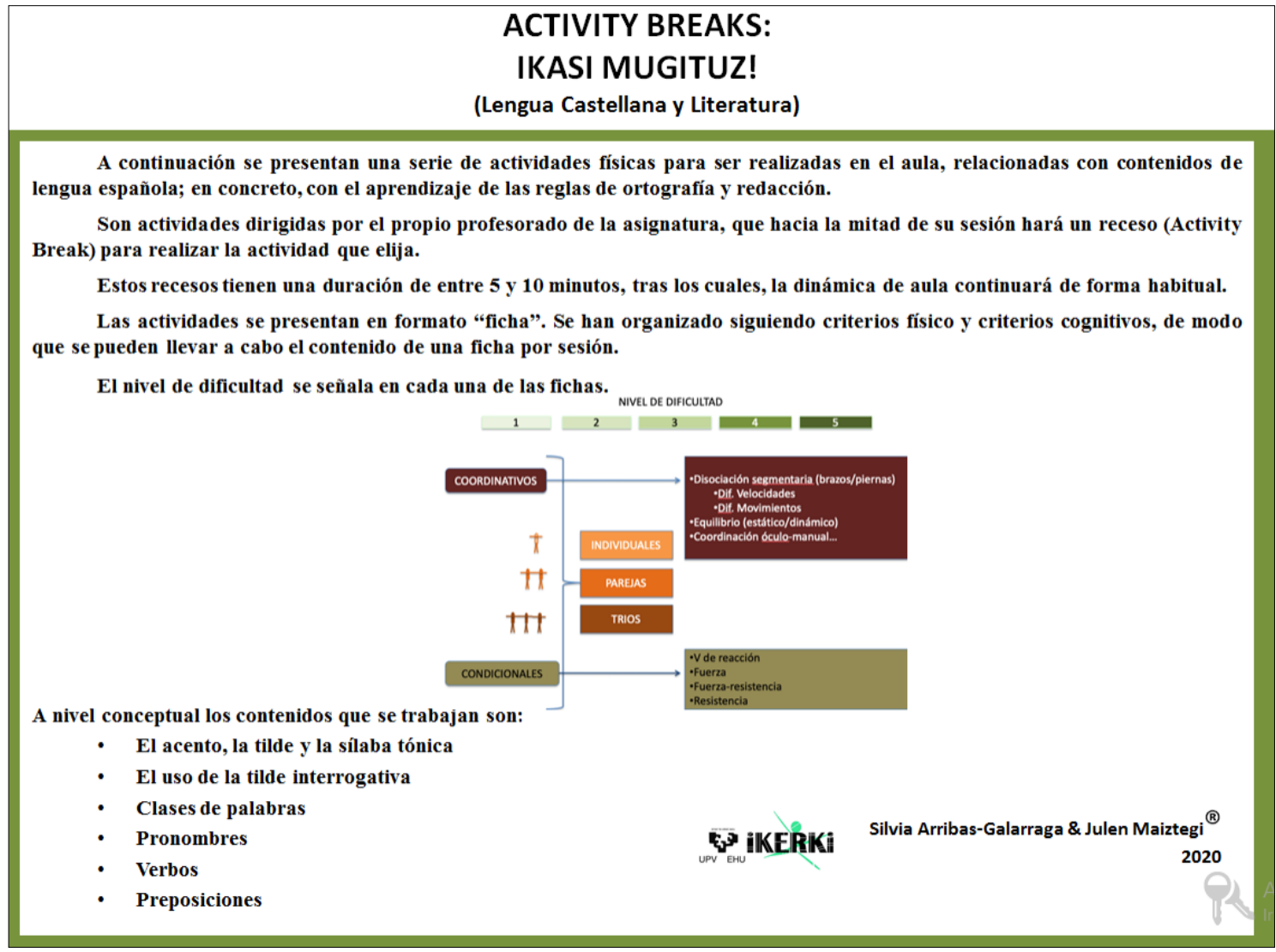

Previamente a la intervención, la profesora realizó un dictado que marcaría el resultado académico previo de cada estudiante, tanto del grupo control como del experimental. La intervención se basó en implementar un programa centrado en descansos activos o Activity Breaks, con la finalidad de activar físicamente al alumnado, e interiorizar y repasar contenidos 
académicos de la asignatura Lengua Castellana y Literatura, del primer curso de la ESO. La implementación del programa de Activity Breaks tuvo lugar durante 7 semanas, con una frecuencia de 4 sesiones por semana y una duración de entre 5 y 10 minutos por sesión.

Aproximadamente hacia la mitad de la sesión de Lengua Castellana y Literatura la profesora (sin preparación específica relacionada con la educación física), indicaba al alumnado el comienzo de la actividad. Escogía una de las fichas de actividades, en función del contenido que quisiera trabajar, y dirigía los descansos activos durante no más de 10 minutos. Transcurrido el tiempo, el grupo volvía a su actividad cotidiana.

\section{Figura 2.}

Ficha de actividad física para repasar las tildes

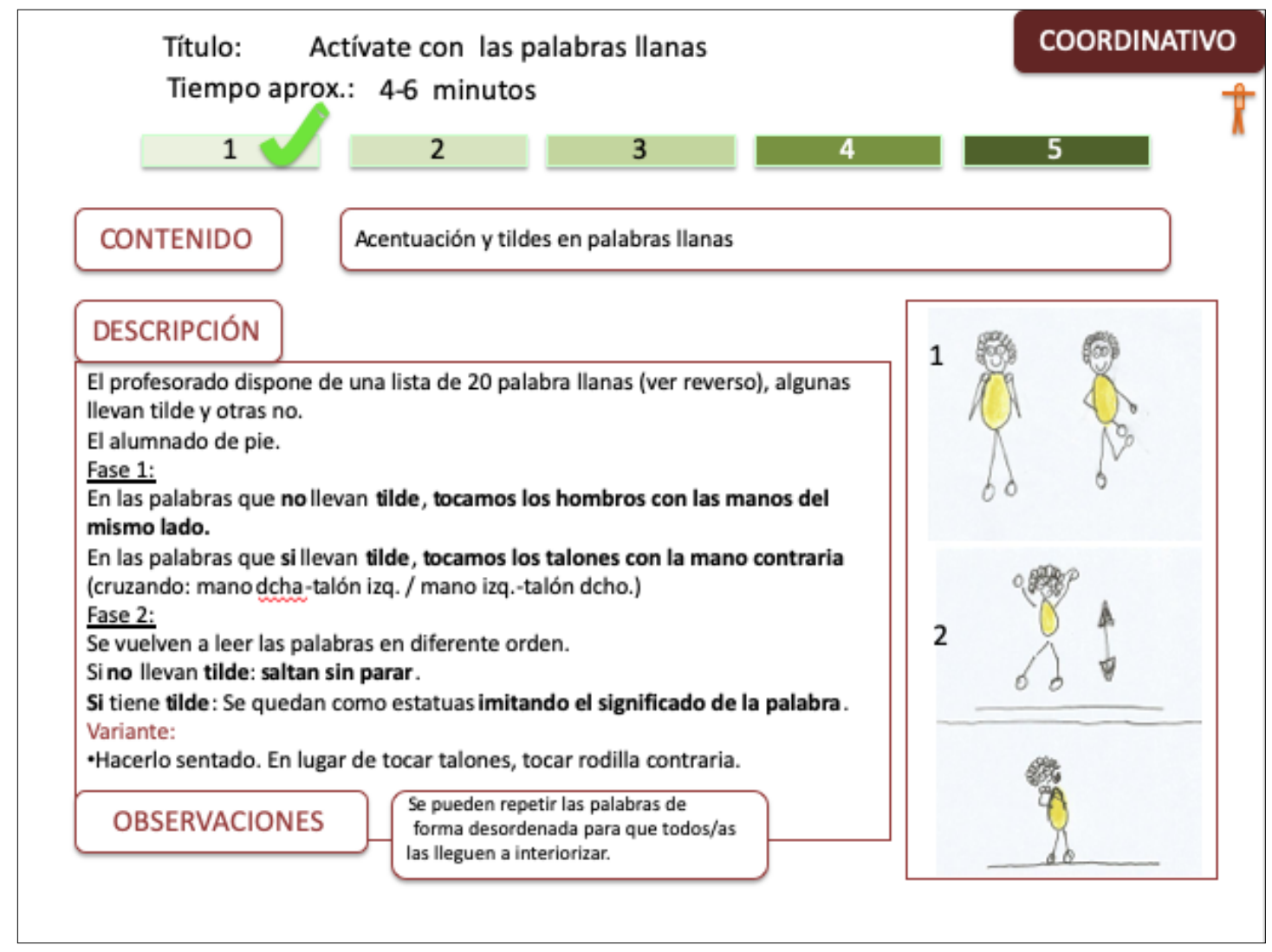

En cuanto a la toma de datos, se llevó a cabo en tres fases: 1) Fase de pretest: con toma de datos en los dos grupo clase; 2) Fase de intervención: tuvo lugar durante 7 semanas en un único grupo (experimental); 3) Fase postest: se realizó tras la intervención en uno de los grupos, y supuso la recogida de datos en ambos grupos.

Asimismo, la profesora realizó el mismo dictado que había realizado antes del inicio de la intervención, con el objetivo de observar los posibles cambios a nivel académico. 
Figura 3.

Listado de palabras para ser identificadas a través de una acción física

\begin{tabular}{|c|c|}
\hline \multicolumn{2}{|c|}{ Actívate con las palabras llanas } \\
\hline & LISTA DE 20 PALABRAS LLANAS \\
\hline 1. Acentúas & 11. Seismo \\
\hline 2. Cáliz & 12. Cártel \\
\hline 3. Río & 13. Búnker \\
\hline 4. Estrellas & 14. Revista \\
\hline 5. Dúo & 15. Temperatura \\
\hline 6. Ladrillo & 16. Néctar \\
\hline 7. Banquero & 17. Púgil \\
\hline 8. Fueron & 18. Canciones \\
\hline 9. Dormía & 19. Abrigo \\
\hline 10. Lluvia & 20. Ágil \\
\hline
\end{tabular}

\section{Análisis de datos}

Para el análisis de los daros se realizaron estadísticos descriptivos, para obtener medias y desviaciones típicas.

El contraste de hipótesis se realizó empleando la prueba no paramétrica de Wilconox, dado que la distribución de datos no cumplía el principio de normalidad.

Para la comparación entre distintos grupos se aplico la prueba U de Mann Whitney.

Todos los daros se trataron por medio del programa informático IBM SPSS Statistics 24.

\section{Resultados}

Teniendo en cuenta el objetivo, analizar y comprobar la evolución de los niveles de atención y concentración en relación a la práctica de descansos activos, se estudiaron los resultados obtenidos en el Test de Atención D2 (Brickenkamp 2002; Cubero, 2012).

Los resultados obtenidos en la fase previa mostraron que la atención en el grupo control era superior en 57.29 puntos promedio respecto a la del grupo experimental. Al comparar los valores iniciales entre el grupo experimental y el grupo control, no se obtuvieron diferencias estadísticamente significativas $(Z=-1.838, p=.066)$, se comprobó la igualdad entre grupos (tabla 1).

En cuanto a la evolución del nivel de atención, la variación de la media obtenida por el grupo experimental aumentó en 64.56 puntos promedio frente al incremento de 48.97 puntos promedio del grupo control. Mediante las pruebas no paramétricas, se confirmó la existencia de diferencias significativas entre los datos pre y post de la variable atención en el grupo experimental $(Z=-3.361, p=.001)$ y también en el grupo control $(Z=-2.983, p=.003)$.

Tras la intervención, comparando el incremento de los niveles de atención entre los dos grupos, no se observaron diferencias estadísticamente significativas en el incremento 
obtenido por el grupo experimental respecto al obtenido por el grupo control $(Z=-0.372, p$ $=.71)$.

Tabla 1.

Puntuaciones medias de atención pretest y postest en función de grupo clase (Rango $0-685)$

\begin{tabular}{|c|c|c|c|}
\hline Grupo & & Pre & Post \\
\hline \multirow{4}{*}{ Control } & $M$ & 360.17 & 409.14 \\
\hline & DT & $(54.19)$ & $(92.56)$ \\
\hline & $z$ & \multicolumn{2}{|c|}{-2.983} \\
\hline & $p$ & \multicolumn{2}{|c|}{$.003^{* *}$} \\
\hline \multirow{4}{*}{ Experimental } & $M$ & 302.88 & 367.44 \\
\hline & DT & $(86.82)$ & $(105.62)$ \\
\hline & $z$ & \multicolumn{2}{|c|}{-3.361} \\
\hline & $p$ & \multicolumn{2}{|c|}{$.001 * * *$} \\
\hline
\end{tabular}

Los datos referentes a la variable concentración en la fase pretest, señalan niveles superiores en 29.63 puntos promedio a favor del grupo control (tabla 2). Mediante los análisis no paramétricos, se comprobó la igualdad entre ambos grupos clase al no haber diferencias estadísticamente significativas entre los valores iniciales del grupo control y grupo experimental $(Z=-1.75, \mathrm{p}=.08)$.

Tras la realización de la intervención mediante los descansos activos, el grupo experimental mostro un mayor aumento (8.01 puntos) respecto al grupo control. Así, los valores de concentración del grupo experimental incrementaron en 31.69 puntos promedio y los del grupo control en 23.68 puntos promedio. Las pruebas no paramétricas confirmaron diferencias estadísticamente significativas entre el pre y post en el grupo experimental $(Z=-$ 2.947, $\mathrm{p}=.003)$ y en el grupo control $(Z=-2.983, \mathrm{p}=.003)$.

Una vez finalizada la fase de intervención, la diferencia de incremento del nivel de concentración del grupo experimental en comparación con el del grupo control, no resulto ser estadísticamente significativa $(Z=-0.279, \mathrm{p}=.78)$.

Tabla 2.

Puntuaciones medias de concentración pretest y postest en función de grupos clase (Rango 0-299)

\begin{tabular}{ccccc}
\hline Grupo & & Pre & Post \\
\hline \multirow{5}{*}{ Control } & $M$ & 139.75 & & 163.43 \\
& $D T$ & $(31.6)$ & & $(45.4)$ \\
& $z$ & & -2.983 & \\
& $p$ & & $.003^{* *}$ & \\
\multirow{5}{*}{ Experimental } & $M$ & 110.12 & & 141.81 \\
& $D T$ & $(47.91)$ & & $(53.21)$ \\
& $z$ & & -2.947 & \\
& $p$ & \multicolumn{3}{c}{$.003^{* *}$} \\
\hline
\end{tabular}

${ }^{*} \mathrm{p}<.05 * * \mathrm{p}<.01 * * * \mathrm{p}<.001$

El segundo objetivo consistía en examinar la relación existente entre el rendimiento académico y los descansos activos. En la tabla 3 se muestran los resultados correspondientes 
al resultado de la prueba académica en la que se reflejaba el aprendizaje de los contenidos obtenidos a través de una prueba de dictado. La tabla muestra los errores cometidos por el grupo control y experimental en los dictados previos y posteriores a la intervención.

Tabla 3.

Puntuaciones medias de los errores realizados en los dictados pre y post

\begin{tabular}{ccccc}
\hline Grupo & & Pre & Post \\
\hline \multirow{5}{*}{ Control } & $M$ & 12.18 & & 11.79 \\
& $D T$ & $(8.02)$ & & $(5.06)$ \\
& $z$ & & -1.577 & \\
& $p$ & & .115 & \\
Experimental & $M$ & 10.24 & & 11.12 \\
& $D T$ & $(7.27)$ & & $(7.17)$ \\
& $z$ & & -0.93 & \\
\hline & $p$ & & .352 & \\
\hline
\end{tabular}

${ }^{*} \mathrm{p}<.05 * * \mathrm{p}<.01 * * * \mathrm{p}<.001$

Como se puede observar, los datos del dictado previo mostraron mejores resultados (menos fallos) en el grupo experimental $(M=10,24)$, que el grupo control $(M=12,18)$. Pero, no se observó diferencia significativa $(Z=-0.495, p=.62)$. Tras la intervención, el número de errores del grupo control disminuyo $(M=11,79)$ en 0.39 puntos promedios. Sin embargo, el número de fallos del grupo experimental $(M=11,12)$ aumentó en 0.88 errores. Las diferencias del pre al post del grupo control $(Z=-1.577, p=.115)$ y del grupo experimental $(Z=-0.93, p=.352)$ no resultaron significativas.

\section{Discusión}

En un contexto en el que, tal y como indica la OMS (2020), los niveles de actividad físicodeportiva de la población mundial son insuficientes tanto en la población adulta, como en la población de menor edad (García Maramoros, 2019), la presente investigación, con el fin, por un lado de reducir los tiempos prolongados en situación sedentaria (Webster, Russ, Vazou, Goh y Erwin, 2015; Méndez-Giménez, 2020; Merriman et al., 2020); y por otro, de profundizar en los estudios que indican que la actividad física podría relacionarse, entre otros, con beneficios en la salud cognitiva (Ahumada Tello y Toffoletto, 2020; Contreras-Jordán et al., 2020; Poitras et al., 2016), introdujo descansos activos o Activity Breaks, en las sesiones de aula de la asignatura Lengua Castellana y Literatura de $1^{\circ}$ de la ESO (Enseñanza Secundaria Obligatoria) de un centro de la Comunidad Autónoma Vasca (España).

El primer objetivo consistió en analizar y comprobar la evolución del nivel de atención y concentración del alumnado en función del programa de descansos activos. Los resultados obtenidos muestran que, tras la intervención, el grupo experimental aumentó tanto el nivel de atención como el nivel de concentración de manera estadísticamente significativa. Estos resultados se sitúan en línea con los estudios llevados a cabo por Pinto-Escalona y Martínezde-Quel (2019), Contreras-Jordan et al. (2020), Ruiz-Ariza et al. (2020) y Janssen et al. (2014) quienes también observaron los beneficios de los descansos activos en los niveles de atención y concentración. Además, se puede afirmar que, mediante los descansos activos, junto al incremento de los niveles de atención y concentración, se ha logrado disminuir los 
tiempos prolongados sedentarios (Webster, Russ, Vazou, Goh y Erwin, 2015; MéndezGiménez, 2020; Merriman et al., 2020) y activar físicamente al alumnado.

En cuanto al segundo objetivo, los resultados de esta investigación, no permiten verificar la existencia de relación entre el rendimiento académico y los descansos activos. Paradójicamente, los resultados del grupo experimental, tras la intervención resultaron menos satisfactorios en la fase post que en la fase inicial. Esto suscita la necesidad de profundizar en este punto. En este sentido resulta relevante el estudio de Tomporowski et al. (2015), quienes afirman que la relación existente entre la cognición y el rendimiento académico es compleja y posiblemente influenciada por otros factores. En la misma línea, otros autores (Kapur, 2018; Willcox, 2011; Torres y Rodríguez, 2006; Zuluaga, 2017; Lamas, 2015) resaltan el carácter multifactorial del rendimiento académico.

Dados los resultados, coincidimos con Álvarez-Bueno et al. (2017) quienes afirman que la actividad físico-deportiva en el aula, se convierte en un factor positivo para desarrollar la cognición de las alumnas y los alumnos, pues al igual que en estudios previos, ayudó a favorecer el incremento significativo de las variables fundamentales en el proceso de aprendizaje, es decir, la atención y concentración (Vanhelst et al., 2016; Méndez-Giménez, 2020; Ruiz-Ariza et al., 2020). Coincidimos asimismo con autores que indican que los descansos activos o Activity Breaks permiten reducir los tiempos prolongados sedentarios (Muñoz-Parreño et al., 2020), aumentar los niveles de práctica de actividad físico-deportiva, incrementando los niveles de actividad moderada o vigorosa (Masini et al., 2020), y así, impulsar un mayor gasto energético de la población infantil y adolescente (Stewart et al, 2004).

Una de las limitaciones de la presente investigación es el número reducido de participantes, no obstante, debido al incremento estadísticamente significativo de los niveles de atención y concentración tras la implementación de los descansos activos o Activity Breaks, se considera interesante seguir investigando en la misma línea, si bien se debería abordar el rendimiento académico desde una perspectiva multifactorial, en la que se tomaran en cuenta factores personales y contextuales.

Para concluir, destacar que los datos aquí mostrados, marcan una interesante tendencia que requieren estudios en profundidad para verificar la posible relación de la actividad física con el rendimiento académico.

\section{Referencias}

Abi, P., Hilberg, E., Schuna, J., John, D., y Gunter, K. (2018). Teacher-Level Factors, Classroom Physical Activity Opportunities, and Children's Physical Activity Levels. Journal of Physical Activity and Health, 15(9), 637-643. doi: doi.org/10.1123/jpah.2017-0218

Ahumada Tello, J., y Toffoletto, M. C. (2020). Factores asociados al sedentarismo e inactividad física en Chile: una revisión sistemática cualitativa. Revista médica de Chile, 148(2), 233241.

Álvarez-Bueno, C., Pesce, C., Cavero-Redondo, I., Sánchez-López, M., Martínez- Hortelano, J. A., y Martínez-Vizcaíno, V. (2017). The Effect of Physical Exercise Activity Interventions on Children's Cognition and Metacognition: A Systematic Review and 
Meta-Analysis. Journal of the American Academy of Child \& Adolescent Psychiatry, 56(9), 729-738. doi: doi.org/10.1016/j.jaac.2017.06.012

Arocha Rodulfo, J. I. (2019). Sedentarismo, la enfermedad del siglo XXI. Clínica e Investigación en Arteriosclerosis, 31(5), 233-240.

Bernate, J. A., Fonseca, E. R., \& Cruz, J. E. L. (2020). Sedentarismo y actividad física: Revisión bibliográfica de estrategias desde la educación física y aplicaciones prácticas para niños y adolescentes. EmásF, Revista Digital de Educación Física, 12(67).

Brickenkamp, R. (2002). D2: test de atención. Tea.

Carson, V., Hunter, S., Kuzik, N., Gray, C., Poitras, V., Chaput, J.P., ... Tremblay, M. (2016). Systematic review of sedentary behaviour and health indicators in school-aged children and youth: an update. Applied Physiology, Nutrition, and Metabolism, 41(6), 240-265. doi: doi.org/10.1139/apnm-2015-0630

Contreras-Jordán, O. R., León, M. P., Infantes-Paniagua, A. y Prieto-Ayuso, A. (2020). Efecto de los descansos activos en la atención y concentración de los alumnos de Educación Primaria. Revista interuniversitaria de formación del profesorado, (95), 145-160.

García Matamoros, W. F. (2019). Sedentarismo en niños y adolescentes: Factor de riesgo en aumento. RECIMUNDO, 3(1), 1602-1624.

Glapa, A., Grzesiak, J., Laudanska-Krzeminska, I., Chin, M.K., Edginton, C., Mo, M., y Bronikowski, M. (2018). The Impact of Brain Breaks Classroom-Based Physical Activities on Attitudes toward Physical Activity in Polish School Children in Third to Fifth Grade. International Journal of Environmental Research and Public Health, 15(2). doi: doi.org/10.3390/ijerph15020368

Janssen, M., Chinapaw, M. J. M., Rauh, S.P., Toussaint, H.M., van Mechelen, W., y Verhagen, E. (2014). A short physical activity break from cognitive tasks increases selective attention in primary school children aged 10-11. Mental Health and Physical Activity, 7(3), 129-134. doi: doi.org/10.1016/j.mhpa.2014.07.001

Kapur, D. R. (2018). Factors Influencing the Students Academic Performance in Secondary Schools in India. Research Gate, 1-25.

Lamas, H. (2015). Sobre el rendimiento escolar. Propósitos y Representaciones, 3(1). doi: doi.org/10.20511/pyr2015.v3n1.74

Layne, T., Yli-Piipari, S., y Knox, T. (2020). Physical activity break program to improve elementary students' executive function and mathematics performance. Education 3-13. International Journal of Primary, Elementary and Early Years Education. doi: doi.org/10.1080/03004279.2020.1746820

Mahar, M. (2011). Impact of short bouts of physical activity on attention-to-task in elementary school children. Preventive Medicine, 52, 60-64. doi: doi.org/10.1016/j.ypmed.2011.01.026

Mahar, M., Murphy, S., Rowe, D., Golden, J., Shields, A., y Readeke, T. (2006). Effects of a Classroom-Based Program on Physical Activity and On-Task Behavior. Medicine and science in sports and exercise 38(12), 2086-2094. doi: doi.org/10.1249/01.mss.0000235359.16685.a3

Marchesi, A., y Pérez, E.M. (2003). La comprensión del fracaso escolar. En Marchesi, A., y Hernández Gil, C (coords.) El fracaso escolar. Una perspectiva internacional. Madrid: Alianza. 
Masini, A., Marini, S., Gori, D., Leoni, E., Rochira, A., y Dallolio, L. (2020). Evaluation of schoolbased interventions of active breaks in primary schools: A systematic review and meta-analysis. Journal of Science and Medicine in Sport, 23(4), 377-384. doi: doi.org/10.1016/j.jsams.2019.10.008

Maureira, F., y Flores, E. (2017). Efectos del ejercicio físico sobre la atención: una revisión de los últimos años. Revista de Ciencias de la Actividad Física UCM. 1(18), 73-83.

Méndez-Giménez, A. (2020). Resultados académicos, cognitivos y físicos de dos estrategias para integrar movimiento en el aula: clases activas y descansos activos. SPORT TK: Revista Euroamericana de Ciencias del Deporte, 9(1), 63-74. doi: doi.org/10.6018/sportk.412531

Merriman, W., González-Toro, C.M., y Cherubini, J. (2020). Physical Activity in the Classroom. Kappa Delta Pi Record, 56(4), 164-169. doi: doi.org/10.1080/00228958.2020.1813518

Ministerio de Sanidad, Consumo y Bienestar Social. (2017). Encuesta Nacional de Salud ENSE, España 2017. Serie informes monográficos. Actividad física, descanso y ocio. Recuperado de: https://www.mscbs.gob.es/estadEstudios/estadisticas/encuestaNacional/encuestaN ac2017/ACTIVIDAD_FISICA.pdf

Muñoz-Parreño, J., Belando-Pedreño, N., Torres-Luque, G., y Valero-Valenzuela, A. (2020). Improvements in Physical Activity Levels after the Implementation of an ActiveBreak-Model-Based Program in a Primary School. Sustainability, 12(9). doi: doi.org/10.3390/su12093592

Ocampo, L. (2009). La atención: un proceso psicológico básico. Revista de la Facultad de Psicología Universidad Cooperativa de Colombia, 5(8), 91-100.

Organización Mundial de la Salud. (2020). Actividad física. Recuperado de: https://www.who.int/es/news-room/fact-sheets/detail/physical-activity

Pesce, C., Crova, C., Cereatti, L., Casella, R., y Bellucci, M., (2009). Physical activity and mental performance in preadolescents: Effects of acute exercise on free-recall memory. Mental Health and Physical Activity, 2(1), 16-22. doi: doi.org/10.1016/j.mhpa.2009.02.001

Pinto-Escalona, T., \& Martínez-de-Quel, O. (2019). Ten minutes of interdisciplinary physical activity improve academic performance. Apunts. Educación Física y Deportes, (138), 82-94. doi: dx.doi.org/10.5672/apunts.2014-0983.es.(2019/4).138.07

Poitras, V., Gray, C.E., Borghese, M., Carson, V., Chaput, J. P., Janssen, I., ... y Tremblay, M., (2016). Systematic review of the relationships between objectively measured physical activity and health indicators in school-aged children and youth. Applied Physiology, Nutrition, and Metabolism, 41(6), 197-236. doi: doi.org/10.1139/apnm-2015-0663

Ríos-Lago, M., Muñoz-Céspedes, J. M., y Paúl-Lapedriza, N. (2007). Alteraciones de la atención tras daño cerebral traumático: evaluación y rehabilitación. Revista de Neurología, 44(5), 291-297.

Robinson, L., Stodden, D., Barnett, L., Lopes, V., Logan, L. P., y D’Hondt, E. (2015). Motor Competence and its Effect on Positive Developmental Trajectories of Health. Sport Medicine, 45(9), 1273-1284. doi: doi.org/10.1007/s40279-015-0351-6

Rodríguez Torres, Á. F., Rodríguez Alvear, J. C., Guerrero Gallardo, H. I., Arias Moreno, E. R., Paredes Alvear, A. E., \& Chávez Vaca, V. A. (2020). Beneficios de la actividad física para 
niños y adolescentes en el contexto escolar. Revista Cubana de Medicina General Integral, 36(2).

Romero, T. (2009). Hacia una definición de sedentarismo. Revista Chilena de Cardiología, 28(4), 409-413. http://dx.doi.org/10.4067/S0718-85602009000300014

Ruiz-Ariza, A., López-Serrano, S., Mezcua-Hidalgo, A., Martínez-López, E., y Abu-Helaiel, K. (2020). Efecto agudo de descansos físicamente activos en variables cognitivas y creatividad en Educación Secundaria. Retos, (39), 635-642. doi: doi.org/10.47197/retos.voi39.78591

Sánchez, M., Gutiérrez, A., Ruiz de la Hermosa, A., López, C., y Sanchez, Mª.I. (2017). Proyectos Escolares Saludables. Descansos Activos. Universidad de Castilla-La Mancha. doi: dx.doi.org/10.18239/atenea.04.2017

Stewart, J., Dennison, D., Kohl, H., y Doyle, A. (2004). Exercise Level and Energy Expenditure in the TAKE 10!® In-Class Physical Activity Program. Journal of School Health, 74(10), 397-400. doi: doi.org/10.1111/j.1746-1561.2004.tbo6605.x

Timmons, B., LeBlanc, B. Carson, V., Connor, S., Dillman, C., Janssen, I., ... y Tremblay, S. (2012). Systematic review of physical activity and health in the early years (aged 0-4 years). Applied Physiology Nutrition and Metabolism, 37(4), 773-792. doi: doi.org/10.1139/h2012-070

Tomporowski, P. D., McCullick, B., Pendleton D. M., y Pesce, C. (2015). Exercise and children's cognition: The role of exercise characteristics and a place for metacognition. Journal of Sport and Health Science, 4(1), 47-55. doi: doi.org/10.1016/j.jshs.2014.09.003

Torres, L., y Rodríguez, N. (2006). Rendimiento académico y contexto familiar en estudiantes universitarios. Enseñanza e investigación en psicología, 11(2), 255-270.

Tremblay, M. S., Colley, R. C., Saunders, T. J., Healy, G. N., y Owen, N. (2010). Physiological and health implications of a sedentary lifestyle. Applied Physiology Nutrition ande Metabolism, 35(6), 725-740. doi: doi.org/10.1139/H10-079

Vanhelst, J., Béghin, L., Duhamel, A., Manios, Y., Molnar, D., De Henauw, S., ... Gottrand, F. (2016). Physical activity is associated with attention capacity in adolescents. The Journal of Pediatrics, 168, 126-131. doi: doi.org/10.1016/j.jpeds.2015.09.029

Webster, C. A., Russ, L., Vazou, S., Goh, T. L., y Erwin, H. (2015). Integrating movement in academic classrooms: understanding, applying and advancing the knowledge base. Obesity Reviews, 16(8), 691-701. doi: doi.org/10.1111/obr.12285

Willcox, $M^{\mathrm{a}}$. R. (2011). Factores de riesgo y protección para el rendimiento académico. Un estudio descriptivo en estudiantes de Psicología de una universidad privada. Revista Iberoamericana de Educación, 55(1), 1-9. doi: doi.org/10.35362/rie5512909

Zuluaga, M. N. C. (2017). Factores que influyen en el rendimiento académico del estudiante. Escenarios: empresa y territorio, 5(5), 93-118. 